\title{
Novel therapeutic strategies against cancer and chronic infection by targeting P-selectin glycoprotein ligand-1 as an immune $T$ cells response regulator
}

\author{
Elisa Ramos-Sevillano ${ }^{1}$, Jose Yuste ${ }^{2,3}$ \\ ${ }^{1}$ Sir William Dunn School of Pathology, University of Oxford, Oxford, UK; ${ }^{2}$ National Center for Microbiology, Institute of Health Carlos III, \\ Madrid, Spain; ${ }^{3}$ CIBERES, Madrid, Spain \\ Correspondence to: Dr. Elisa Ramos-Sevillano. Sir William Dunn School of Pathology, University of Oxford, Oxford, UK. Email: elisa.ramos@path.ox.ac.uk; \\ Dr. Jose Yuste. National Center for Microbiology, Institute of Health Carlos III, Madrid, Spain. Email: jyuste@isciii.es.
}

Submitted Aug 24, 2016. Accepted for publication Sep 02, 2016.

doi: $10.21037 /$ tcr.2016.10.28

View this article at: http://dx.doi.org/10.21037/tcr.2016.10.28

P-selectin glycoprotein ligand-1 (PSGL-1) is a homodimeric mucin-like glycoprotein primarily restricted to cells of hematopoietic origin. Although, it may be present on some non-immune cells such as epithelial cells lining the fallopian tube, microvascular endothelial cells, and platelets, PSGL1 is broadly expressed on most immune cells including neutrophils, monocytes, dendritic cells and lymphocytes $(1,2)$. PSGL-1 on leukocytes mediates interactions with $\mathrm{P}$-selectin and E-selectin present in activated endothelium with a major role in leukocyte adhesion and transmigration as it is responsible for the initial steps of the extravasation cascade $(1,2)$. PSGL-1 is also responsible for controlling $\mathrm{T}$ cells trafficking into lymphoid and inflamed tissues conferring to this receptor a pleiotropic nature $(3,4)$. This is important in the context of chronic infection, autoimmune and allergic diseases and also in cancer, where loss of $\mathrm{T}$ cells responsiveness may hinder an effective control of the immune response mediated by $\mathrm{T}$ cells $(5,6)$. Functional polarization of $T$ cells responses contribute to the outcome of certain diseases. In this sense, type 1 immune responses provide beneficial immune protection against acute infections caused by viruses and intracellular bacteria, whereas cytotoxic effects triggered by $\mathrm{CD} 8^{+} \mathrm{T}$ cells and $\mathrm{CD}^{+} \mathrm{T}$ helper $1\left(\mathrm{~T}_{\mathrm{H}} 1\right)$, are critical players of the immune response against cancer $(5,6)$. As these $\mathrm{CD}^{+}$and $\mathrm{CD} 4^{+}$ $\mathrm{T}_{\mathrm{H}} 1$ cells display antitumor activity, downregulation of $\mathrm{T}$ cells population is an impediment to control and eliminate cancer cells.

Chronic viral infection may induce $\mathrm{T}$ cells dysfunction characterized by reduced proliferation, impaired cytokine production and altered co-stimulatory and inhibitory signals due to repetitive antigen stimulation (7). Dysfunctional $\mathrm{T}$ cells can also be found in cancer as this is a dynamic antigen-driven differentiation event during tumor development, facilitating cancer growth (8). The dysfunctional state of $\mathrm{T}$ cells is commonly called exhaustion, on the basis of similarities to dysfunctional $\mathrm{T}$ cells in chronic infections. The majority of our knowledge about $\mathrm{CD}^{+} \mathrm{T}$ cells exhaustion is derived from models of chronic infections in mice, normally using the lymphocytic choriomeningitis virus (LCMV) infection model $(9,10)$. In comparison to chronic infections, T cells dysfunction in cancer has genetic differences that may be explained by the diversity of the microenvironments $(11,12)$. In a recent study by Tinoco et al., they found that PSGL-1 located on the surface of $\mathrm{T}$ cells, acts as a negative regulator of $\mathrm{T}$ cells function (Figure 1). These authors demonstrate that PSGL-1 has the capacity to dampen T cells signals promoting the exhaustion of $\mathrm{T}$ cells through the induction of multiple immune checkpoint receptors (13). PSGL-1 was highly expressed on T cells after chronic viral infection with LCMV. Using mice lacking PSGL-1, they found that virus-specific $\mathrm{T}$ cells were preserved in increased numbers resulting in enhanced survival and reduced expression of inhibitory receptors including programme cell death protein 1 (PD-1). This is important in terms of disease, as T cells exhaustion is commonly linked to expression of multiple inhibitory molecules that can affect survival after viral infection $(14,15)$. In the absence of PSGL-1, reduced viral titers of LCMV were found although increased mortality 

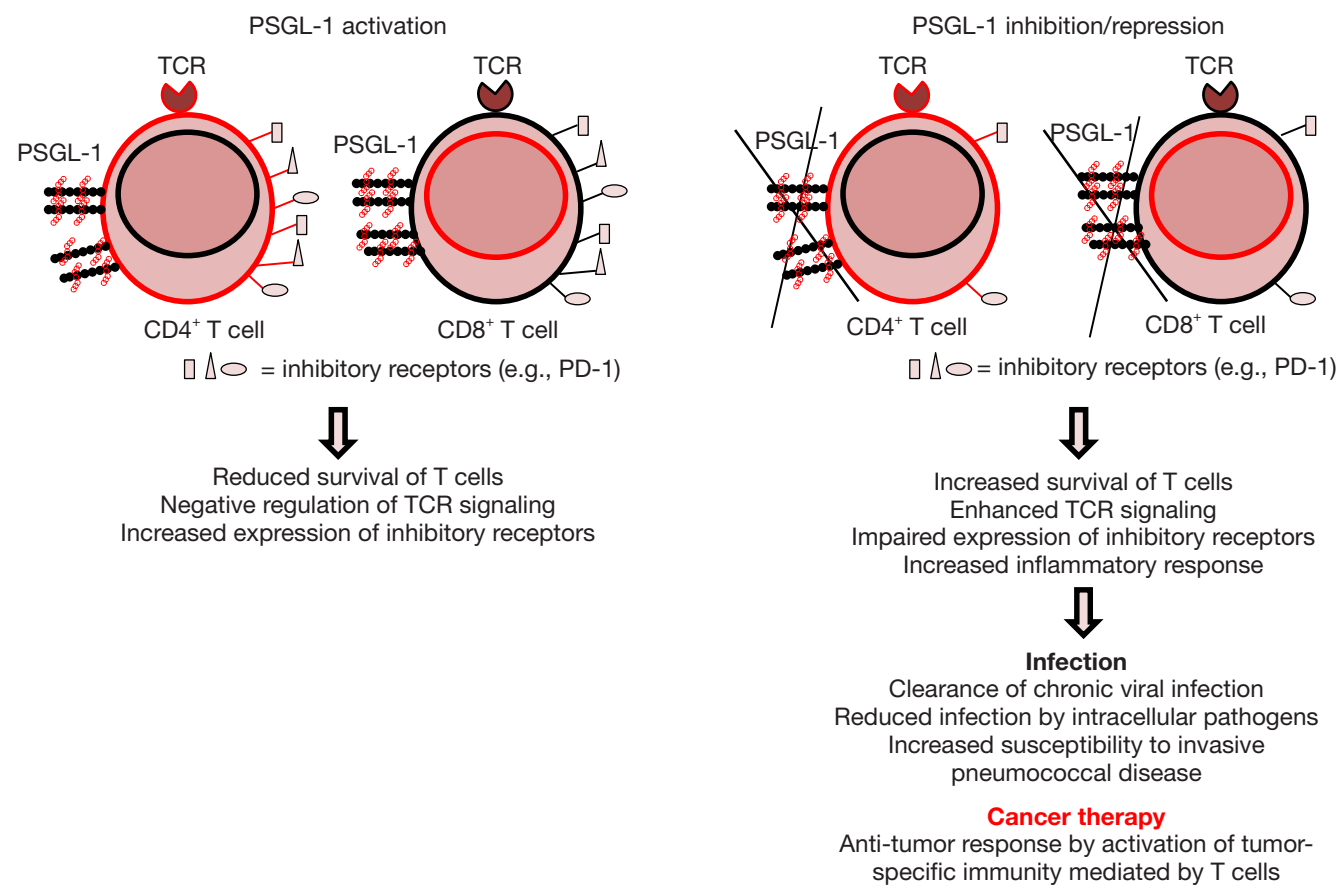

Figure 1 Schematic representation of the impact of PSGL-1 activation or inhibition in terms of host immune response mediated by T cells and the consequences for infectious diseases and cancer therapy. PSGL-1, P-selectin glycoprotein 1 ligand; TCR, T cell receptor; PD-1, programme cell death protein 1.

was also observed due to elevated levels of pro-inflammatory cytokines associated to increased inflammation and tissue damage (13). In addition, $\mathrm{CD}^{+}$and $\mathrm{CD}^{+} \mathrm{T}$ cells survival was improved after LCMV infection when PSGL-1 is missing. This is consistent with an acute pneumonia model of infection caused by Streptococcus pneumoniae as T cells counts were higher in mice lacking PSGL-1. During pneumonia, PSGL-1 controls the severity of pneumococcal dissemination from the lung to the bloodstream. In systemic infection, a major role of PSGL-1 in host defense is to clear the bacteria in the systemic circulation controlling bacterial replication (16).

In the context of $\mathrm{T}$ cell receptor (TCR) stimulation, Bradley and colleagues, demonstrated that PSGL-1 negatively regulates TCR signaling observing an increased expression of inhibitory receptors such as PD-1, limiting the survival of $\mathrm{CD}^{+} \mathrm{T}$ cells, which exacerbated $\mathrm{T}$ cells exhaustion (Figure 1) (13). This kind of response is very relevant in the cancer field, as tumor-infiltrating lymphocytes linked to progressing tumors and metastatic lesions, express high levels of inhibitory receptors including PD-1 $(17,18)$. In the absence of PSGL-1, CD $8^{+} \mathrm{T}$ cells have reduced inhibitory receptor expression which may be a useful immunotherapeutic target against cancer (13). In this sense, chemotherapy using specific monoclonal antibodies that hamper the interaction of certain inhibitor receptors such as $\mathrm{PD}-1$, can improve anti-tumor $\mathrm{T}$ cells responses and produce tumor regression, especially in patients with advanced melanoma $(19,20)$. The study by Tinoco et al. is relevant from the anti-cancer perspective, as PSGL-1 limits anti-tumor responses by promoting high levels of PD-1 expression and loss of multifunctional effectors, whereas in the absence of PSGL-1, tumor control is enhanced (Figure 1). These findings were obtained by injecting melanoma tumor cells in wild-type mice and null mice in PSGL-1 observing that in the absence of PSGL-1 a significant proportion of mice did not develop melanoma whereas all the wild-type mice had tumors. The numbers of tumor infiltrating $\mathrm{CD} 44^{\text {hi }}$ effector $\mathrm{CD}^{+}$and $\mathrm{CD} 4^{+} \mathrm{T}$ cells were significantly increased in mice lacking PSGL-1 and the expression of PD-1 on both $\mathrm{CD}^{+}$and $\mathrm{CD} 8^{+} \mathrm{T}$ cells was lower in the absence of PSGL-1 (13). This study demonstrates independent roles of PSGL-1 in the immune regulation of $\mathrm{T}$ cells responses against cancer and also against chronic viral infection. The results by Tinoco et al., confirm that PSGL-1 plays a key role as a negative 
regulator of the $T$ cells response that can be used by certain virus associated to persistent infection, to control effector $\mathrm{T}$ cells, producing chronical disease. Interestingly, PSGL-1 also represses $\mathrm{T}$ cells responses in the context of tumor development, demonstrating the broad activity of this cellular ligand in the regulation of the immune response.

Overall, the relevance of these findings may be of great interest for pharmaceutical companies in order to explore the potential benefits of targeting the regulator PSGL-1. Activation of PSGL-1 in order to inhibit T cells immune responses may be a useful therapy against autoimmune diseases including multiple sclerosis, lupus, rheumatoid arthritis and psoriasis in which $\mathrm{T}$ cells responses are known to be dysregulated. As PSGL-1 is a receptor on leukocytes involved in the recognition and clearance of $S$. pneumoniae from the systemic circulation, therapies triggering PSGL-1 expression could potentially be useful against pneumonia and invasive disease caused by this devastating human pathogen (16). Inhibition of PSGL-1 can also be useful to avoid the infection process caused by certain intracellular pathogens using this receptor to enter eukaryotic cells and replicate intracellularly (16). This is the case of enterovirus 71 , Anaplasma phagocytophilum or Ebrlichia spp., causing hand-foot-mouth disease and granulocytic anaplasmosis/ ehrlichiosis respectively. As lack of PSGL-1 provides immunity against chronic viral infection and specific tumors by reducing several T-cell inhibitory receptors, this strategy may contribute to the development of novel treatments against these diseases. There is increased evidence that the combination of drugs triggering $\mathrm{T}$ cells responses and inhibitors of immune checkpoint regulators induce powerful and efficient antitumor immune effects, providing broad opportunities for clinical development. In this context, PSGL-1 inhibitors could be a novel therapeutic approach and a promising weapon for patients affected by cancer who are not responding efficiently to currently available immune checkpoints inhibitors. In addition, combination of PSGL1 inhibitors with other immune therapies targeting different immune checkpoint regulators, might offer synergistic alternatives for patients with cancer or chronic viral infections such as hepatitis, than the use of either approach alone. Mechanisms of $T$ cells inhibition remain of considerable importance for immunotherapy, and a better understanding of the natural mechanisms of $\mathrm{T}$ cells regulation will provide further therapeutic interventions. Although, further investigation is required to extend the relevance of PSGL1 as key immune checkpoint regulator, the study by Bradley and colleagues open a promising potential novel anti-cancer therapeutic line by combining PSGL-1 inhibition with immune checkpoint regulators of $\mathrm{T}$ cells dysfunction.

\section{Acknowledgements}

Funding: Dr. Yuste's lab is supported by Ministerio de Economía y Competitividad (grant SAF2012-39444-C02) and by Centro de Investigación Biomédica en Red (CIBERES).

\section{Footnote}

Provenance: This is a Guest Commentary commissioned by Section Editor Chen Qian (Center for Inflammation \& Epigenetics, Houston Methodist Hospital Research Institute, Houston, USA).

Conflicts of Interest: The authors have no conflicts of interest to declare.

Comment on: Tinoco R, Carrette F, Barraza ML, et al. PSGL-1 Is an Immune Checkpoint Regulator that Promotes T Cell Exhaustion. Immunity 2016;44:1190-203.

\section{References}

1. Moore KL, Stults NL, Diaz S, et al. Identification of a specific glycoprotein ligand for P-selectin (CD62) on myeloid cells. J Cell Biol 1992;118:445-56.

2. McEver RP, Cummings RD. Role of PSGL-1 binding to selectins in leukocyte recruitment. J Clin Invest 1997;100:S97-103.

3. Veerman KM, Williams MJ, Uchimura K, et al. Interaction of the selectin ligand PSGL-1 with chemokines CCL21 and CCL19 facilitates efficient homing of T cells to secondary lymphoid organs. Nat Immunol 2007;8:532-9.

4. Haddad W, Cooper CJ, Zhang Z, et al. P-selectin and P-selectin glycoprotein ligand 1 are major determinants for Th1 cell recruitment to nonlymphoid effector sites in the intestinal lamina propria. J Exp Med 2003;198:369-77.

5. Kaech SM, Cui W. Transcriptional control of effector and memory $\mathrm{CD}^{+} \mathrm{T}$ cell differentiation. Nat Rev Immunol 2012;12:749-61.

6. Smyth MJ, Dunn GP, Schreiber RD. Cancer immunosurveillance and immunoediting: the roles of immunity in suppressing tumor development and shaping tumor immunogenicity. Adv Immunol 2006;90:1-50.

7. Speiser DE, Ho PC, Verdeil G. Regulatory circuits of T cell function in cancer. Nat Rev Immunol 2016;16:599-611. 
8. Schietinger A, Philip M, Krisnawan VE, et al. TumorSpecific T Cell Dysfunction Is a Dynamic AntigenDriven Differentiation Program Initiated Early during Tumorigenesis. Immunity 2016;45:389-401.

9. Barber DL, Wherry EJ, Masopust D, et al. Restoring function in exhausted CD8 T cells during chronic viral infection. Nature 2006;439:682-7.

10. Kaech SM, Wherry EJ. Heterogeneity and cellfate decisions in effector and memory $\mathrm{CD}^{+} \mathrm{T}$ cell differentiation during viral infection. Immunity 2007;27:393-405.

11. Baitsch L, Baumgaertner P, Devêvre E, et al. Exhaustion of tumor-specific CD8+ T cells in metastases from melanoma patients. J Clin Invest 2011;121:2350-60.

12. Giordano M, Henin C, Maurizio J, et al. Molecular profiling of CD8 $\mathrm{T}$ cells in autochthonous melanoma identifies Maf as driver of exhaustion. EMBO J 2015;34:2042-58.

13. Tinoco R, Carrette F, Barraza ML, et al. PSGL-1 Is an Immune Checkpoint Regulator that Promotes T Cell Exhaustion. Immunity 2016;44:1190-203.

14. Blackburn SD, Crawford A, Shin H, et al. Tissue-specific differences in PD-1 and PD-L1 expression during chronic viral infection: implications for CD8 T-cell exhaustion. J Virol 2010;84:2078-89.

15. Petrovas C, Casazza JP, Brenchley JM, et al. PD-1 is a regulator of virus-specific $\mathrm{CD}^{+} \mathrm{T}$ cell survival in $\mathrm{HIV}$ infection. J Exp Med 2006;203:2281-92.

16. Ramos-Sevillano E, Urzainqui A, de Andrés B, et al. PSGL-1 on Leukocytes is a Critical Component of the Host Immune Response against Invasive Pneumococcal Disease. PLoS Pathog 2016;12:e1005500.

17. Ostrand-Rosenberg S, Horn LA, Haile ST. The programmed death-1 immune-suppressive pathway: barrier to antitumor immunity. J Immunol 2014;193:3835-41.

18. Gros A, Robbins PF, Yao X, et al. PD-1 identifies the patient-specific CD8+ tumor-reactive repertoire infiltrating human tumors. J Clin Invest 2014;124:2246-59.

19. Page DB, Postow MA, Callahan MK, et al. Immune modulation in cancer with antibodies. Annu Rev Med 2014;65:185-202.

20. Wolchok JD, Kluger H, Callahan MK, et al. Nivolumab plus ipilimumab in advanced melanoma. N Engl J Med 2013;369:122-33.
Cite this article as: Ramos-Sevillano E, Yuste J. Novel therapeutic strategies against cancer and chronic infection by targeting P-selectin glycoprotein ligand-1 as an immune $\mathrm{T}$ cell response regulator. Transl Cancer Res 2016;5(Suppl 4):S868S871. doi: $10.21037 /$ tcr.2016.10.28 